\title{
Quantitative resuscitation in sepsis
}

\author{
Reviewed by: Catherine Patocka, MDCM; ${ }^{*}$ JoelTurner, $\mathrm{MDCM}^{\dagger}{ }^{\dagger}$ Eddy Lang, $\mathrm{MDCM}^{\dagger}$
}

\begin{abstract}
Clinical question
Does a quantitative resuscitation strategy improve mortality from severe sepsis?

Article chosen

Jones AE, Brown MD, Trzeciak S, et al. The effect of a quantitative resuscitation strategy on mortality in patients with sepsis: a meta-analysis. Crit Care Med 2008;36:2932-3.

\section{Study objective}

The authors sought to determine whether quantitative resuscitation (structured cardiovascular intervention with intravascular volume expansion and vasoactive agent support to achieve explicit predefined physiologic end points) improves mortality in severe sepsis and whether the timing of this resuscitation impacts mortality.
\end{abstract}

\section{BACKGROUND}

Severe sepsis and septic shock are significant sources of morbidity and mortality in the emergency department (ED), with in-hospital mortality rates from sepsis remaining virtually unchanged between 1970 and $2000 .^{1-3}$ In 2001, Rivers and colleagues ${ }^{4}$ demonstrated a $16 \%$ absolute reduction in mortality in patients with severe sepsis treated with a protocol-driven resuscitation strategy aimed at hemodynamic optimization in the ED. Their strategy, termed "early goal-directed therapy" (EGDT), used an algorithmic approach to achieve specific resuscitation end points.

In 2008, the Surviving Sepsis Campaign, a conglomeration of physicians from multiple specialties endorsed by 11 societies, updated their guidelines to recommend that such a quantitative resuscitation strategy be implemented at the time of recognition of severe sepsis. ${ }^{5}$ This particular recommendation has met with significant controversy because it is heavily weighted on one single-centre trial. ${ }^{6-8}$

Quantitative resuscitation, as seen in the study by Rivers and coauthors, ${ }^{4}$ involves structured cardiovascular intervention with intravascular volume expansion and vasoactive agent support to achieve explicit predefined physiologic end points using measurements and samples from invasive central venous and arterial monitors. A significant barrier to the widespread implementation of EGDT has been the use of such invasive monitoring., ${ }^{9} 10$

Also known as "goal-oriented resuscitation," quantitative resuscitation is not new, as clinical trials using some form of it have been performed for 20 years. ${ }^{11}$ Jones and colleagues sought to determine whether the mortality benefit seen in these trials was derived from quantitative resuscitation in general, rather than the choice of specific end points.

\section{STUDY DESIGN AND POPULATION STUDIED}

The study was a systematic review and meta-analysis. The investigators looked at all randomized controlled trials of adult patients with a presumed or confirmed diagnosis of sepsis receiving a structured cardiovascular intervention aimed at achieving predefined hemodynamic end points (Box 1).

Box 1. Inclusion criteria of the subject study

- Randomized controlled trials

- Patients aged $>17 \mathrm{yr}$

- Presumptive or confirmed diagnosis of sepsis

- Experimental study using

intervention consisting of a structured cardiovascular resuscitation protocol administered to achieve predefined hemodynamic end points

a control group that received standard of care therapy

From the *McGill Emergency Medicine Residency Program, Montréal, Que., and the †Emergency Department, Sir Mortimer B. Davis Jewish General Hospital, Montréal, Que.

Submitted Jul. 14, 2009; Revised Oct. 5, 2009; Accepted Oct. 8, 2009

This article has been peer reviewed.

CJEM 2010;12(2):150-3 


\section{OUTCOMES MEASURED}

The primary outcome measured was mortality at the end of a predetermined time frame reported by the individual authors, calculated as an odds ratio (OR) with 95\% confidence intervals (CIs). In-hospital mortality was used preferentially.

The authors also examined a priori planned subgroups of early quantitative resuscitation; that is, therapy implemented at the time of recognition of sepsis or within 24 hours. This is in contrast to late quantitative resuscitation, which refers to therapy initiated after 24 hours, or unknown or unreported timing.

A sensitivity analysis was conducted to determine whether including only high-quality randomized controlled trials would yield different results. Studies reporting adequate concealment of allocation (grade A) were considered "high quality," and studies reporting unclear or inadequate concealment of allocation were considered grades B and C, respectively.

Post hoc analysis was done to determine whether any individual study was exerting undue influence over the results.

\section{RESULTS}

An extensive literature search yielded 903 relevant articles. Two independent reviewers screened the titles for eligibility. Their blinded interobserver agreement was 98.7\% ( $\kappa=0.70)$. Twenty-nine titles met the inclusion criteria. Following review of each manuscript by 3 independent reviewers, 9 studies remained, providing a total sample size of 1001.

The summary OR of 0.64 (95\% CI 0.43 to 0.96 ) demonstrated a potentially significant reduction in mortality; however, the 9 studies demonstrated statistical heterogeneity ( $p=0.07, I^{2}=45 \%$ ) and clinical heterogeneity (specifically in the timing of their intervention).
Funnel plot and Egger regression analysis did not suggest the presence of publication bias (intercept $=$ $0.61,95 \% \mathrm{CI}-2.4$ to $3.6, p=0.65$ )

In the a priori defined subgroups of early versus late intervention, patients randomly assigned to receive early quantitative resuscitation compared with standard resuscitation had a significantly lower mortality rate of $39 \%$ (OR 0.50, 95\% CI 0.37 to 0.69) compared with the control group mortality rate of $57 \%$. The 6 early quantitative resuscitation studies included in this subgroup showed minimal heterogeneity $\left(p=0.40, I^{2}=\right.$ $2.4 \%)$. It did not appear that the lower quality studies (grades B and C) were biasing the effect size as the sensitivity analysis yielded consistent findings.

Patients randomly assigned to the late quantitative resuscitation strategy did not have a significantly different mortality rate compared with standard resuscitation (OR 1.16, 95\% CI 0.60 to 2.22).

The authors concluded that quantitative resuscitation provided a survival benefit if instituted early, a benefit that was lost if instituted late.

\section{COMMENTARY}

The meta-analysis by Jones and colleagues fulfilled all the methodological criteria for a high-quality systematic review and meta-analysis as defined by the Quality of Reporting of Meta-analyses (QUORUM) conference. ${ }^{12}$ Specifically, the researchers conducted a thorough search of the literature. Selection of studies was systematic and reproducible with substantial interobserver agreement among reviewers. A potential flaw of the analysis is that it cannot completely exclude publication bias, specifically the exclusion of small negative trials.

With regard to other methodological quality, allocation of concealment was appropriately graded and no problems with incomplete outcome data or selective reporting biases were identified. Blinding assessors and

\begin{tabular}{|c|c|c|c|c|}
\hline Study & Baseline mortality, \% & Mortality timing & Time to intervention & End point \\
\hline Rivers et al. ${ }^{4}$ & 44 & Hospital & $1.3-1.5 \mathrm{~h}$ & $\mathrm{ScvO}_{2}$ \\
\hline Lin et al. ${ }^{13}$ & 71.2 & $\mathrm{ICU}$ & $<4 \mathrm{~h}$ & CVP, MAP, UO \\
\hline Alia et al. ${ }^{14}$ & 66 & $\mathrm{ICU}$ & $<24 \mathrm{~h}$ after ICU admission & $\mathrm{DO}_{2} \mathrm{I}$ \\
\hline Yu et al. ${ }^{15}$ & 52 & $\mathrm{ICU}$ & $\leq 24 \mathrm{~h}$ of diagnosis or insertion of PAC & $\mathrm{DO}_{2} \mathrm{I}$ \\
\hline Yu et al. ${ }^{16}$ & 27 & 30-day & $\leq 24 \mathrm{~h}$ of diagnosis or insertion of PAC & $\mathrm{DO}_{2} \mathrm{I}$ \\
\hline Tuchschmidt et al. ${ }^{17}$ & 72 & 14-day & $<4 \mathrm{~h}$ & $\mathrm{Cl}$ \\
\hline
\end{tabular}

$\mathrm{Cl}=$ cardiac index; $\mathrm{CVP}=$ central venous pressure; $\mathrm{DO}_{2} \mathrm{I}=$ oxygen delivery index (calculation using cardiac index, hemoglobin, mixed venous and arterial oxygen saturation); $\mathrm{ICU}=$ intensive care unit; $\mathrm{MAP}=$ mean arterial pressure; $\mathrm{PAC}=$ pulmonary artery catheter; $\mathrm{ScvO}_{2}=$ central venous oxygen saturation; $\mathrm{UO}=$ urine output. 
patients of outcomes was not performed in any of the studies; however, the chance that this would significantly influence their primary outcome, mortality, is minimal.

Based on the combined baseline mortalities of the studies in the early subgroup, the number needed to treat is 6 . This value is questionable given that the baseline mortality rates of the included studies were somewhat variable, as demonstrated in Table $1 .{ }^{13-17}$

The implications of this study are in keeping with the Canadian Association of Emergency Physicians Sepsis Guidelines ${ }^{18}$ recommendation for a quantitative resuscitation strategy. The implications are also consistent with the Surviving Sepsis Campaign 2008 Guidelines, ${ }^{5}$ which specifically recommend early goal-directed therapy upon recognition of severe sepsis or septic shock, with the option to substitute mixed venous oxygen saturation in place of central venous oxygen saturation as the end point.

Rivers and coworkers' landmark study of early goaldirected therapy in the ED showed a significant difference in the mortality of patients with severe sepsis. The cost of this mortality benefit was a labour-intensive protocol involving arterial and central venous catheterization and continuous central venous oxygen saturation monitoring for at least 6 hours by ED staff. There may be significant barriers to such a protocol in many EDs that are overstretched and cannot prioritize the specialized tools, staff and training needed for EGDT.

Despite significant variability in the goals and end points targeted by the studies in this meta-analysis, the mortality benefit remained. This suggests that it is not the specific end point that makes the difference but rather the fact that intervention occurs early and that it is focused on achieving a specific quantifiable goal. The implication is that other more realistically achievable quantitative end points may be used to provide a similar mortality benefit.

In applying these results to the ED one must be cautious in interpreting the term "early," which in this meta-analysis was defined as within 24 hours. Further studies are required to determine whether a shorter time frame, such as the 6 hours used in Rivers and colleagues' study of EGDT, will offer additional mortality benefit.

Although it would not obviate the need for central access, preliminary results from the Lactate Assessment in the Treatment of Early Sepsis trial indicate that using central venous lactate may be equivalent to central venous oxygen saturation as an end point. ${ }^{19}$ Even more promising is the Protocolized Care for Early Septic Shock trial in which researchers are investigating the use of standard blood pressure and pulse oximetry as end points of resuscitation. ${ }^{20}$

\section{CONCLUSION}

There is clear evidence supporting the value of quantitative resuscitation within 24 hours, including approaches other than EGDT, in the treatment of patients with severe sepsis and septic shock presenting to the ED. The exact goals and end points that must be achieved within the time frame of a patient's stay in the $\mathrm{ED}$ require further delineation.

\section{Competing interests: None declared.}

Keywords: sepsis, quantitative resuscitation, early goaldirected therapy

\section{REFERENCES}

1. Strehlow MC, Emond SD, Shapiro NI, et al. National study of emergency department visits for sepsis, 1992 to 2001. Ann Emerg Med 2006;48:326-31.

2. Angus DCMMF, Linde Zwirble WT, Lidicker JM, et al. Epidemiology of severe sepsis in the United States: analysis of incidence, outcome, and associated costs of care. Crit Care Med 2001;29:1303-10.

3. Kochanek KD, Smith BL. Deaths: preliminary data for 2002. Natl Vital Stat Rep 2004;52:1-47.

4. Rivers E, Nguyen B, Havstad S, et al. Early goal-directed therapy in the treatment of severe sepsis and septic shock. N Engl J Med 2001;345:1368-77.

5. Dellinger RP, Levy MM, Carlet JM, et al. Surviving Sepsis Campaign: international guidelines for management of severe sepsis and septic shock: 2008. Crit Care Med 2008;36:296-327.

6. Ho BC, Bellomo R, McGain F, et al. The incidence and outcome of septic shock patients in the absence of early-goal directed therapy. Crit Care 2006;10:R80.

7. Peake S, Webb S, Delaney A. Early goal-directed therapy of septic shock: We honestly remain skeptical. Crit Care Med 2007;35:994.

8. Sarkar S, Kupfer Y, Tessler S. Goal-directed therapy for severe sepsis. N Engl J Med 2002;346:1025.

9. Carlbom DJ, Rubenfeld GD. Barriers to implementing protocol based sepsis resuscitation in the emergency department results of a national survey. Crit Care Med 2007;35:2525-32.

10. Trzeciak S, Dellinger RP, Abate NL, et al. Translating research to clinical practice: a 1-year experience with implementing early goal-directed therapy for septic shock in the emergency department. Chest 2006;129:225-32. 
11. Shoemaker WC, Appel PL, Kram HB, et al. Prospective trial of supranormal values of survivors as therapeutic goals in high-risk surgical patients. Chest 1988;94:1176-86.

12. Moher D, Cook DJ, Eastwood S, et al.; QUOROM Group. Improving the quality of reports of meta-analyses of randomised controlled trials: the QUOROM statement. Lancet 1999;354:1896-900.

13. Lin SM, Lin HC, Liu CY, et al. A modified goal-directed protocol improves clinical outcomes in intensive care unit patients with septic shock: a randomized controlled trial. Shock 2006;26:551.

14. Alia I, Esteban A, Gordo F, et al. A randomized and controlled trial of the effect of treatment aimed at maximizing oxygen delivery in patients with severe sepsis or septic shock. Chest 1999;115:453-61.

15. Yu M, Burchell S, Hasaniya NWMA, et al. Relationship of mortality to increasing oxygen delivery in patients $>$ or $=$ to 50 years of age: a prospective, randomized trial. Crit Care Med 1998;26:1011.

16. Yu M, Levy MM, Smith P, et al. Effect of maximizing oxygen delivery on morbidity and mortality rates in critically ill patients: a prospective, randomized, controlled study. Crit Care Med 1993;21:830.
17. Tuchschmidt J, Fried J, Astiz M, et al. Elevation of cardiac output and oxygen delivery improves outcome in septic shock. Chest 1992;102:216-20.

18. Green RS, Djogovic D, Gray S, et al. Canadian Association of Emergency Physicians sepsis guidelines: the optimal management of severe sepsis in Canadian emergency departments. CJEM 2008;10:443-59.

19. National Institute of General Medical Sciences (NIGMS). Lactate assessment in the treatment of early sepsis: the LactATES Trial. Bethesda (MD): National Library of Medicine; 2000. Available: http://clinicaltrials.gov/show/NCT00372502 (accessed 2010 Jan 26). NLM Identifier: NCT00372503.

20. National Institute of General Medical Sciences (NIGMS); University of Pittsburgh. Protocolized care for early septic shock (ProCESS). Bethesda (MD): National Library of Medicine; 2000. Available: http://clinicaltrials.gov/show/NCT 00510835 (accessed 2010 Feb 1). NLM Identifier: NCT 00510835 .

Correspondence to: Dr. Catherine Patocka, Emergency Medicine Residency Program, McGill University Health Centre, 687 Pine Ave. W., Montréal QC H3A 1A1; catherine.patocka@mail.mcgill.ca

\section{À LA RECHERCHE D'UN NOUVEL EMPLOI?}

En version imprimée et en ligne, la meilleure façon de placer une annonce ou de trouver un emploi en médecine d'urgence dans un contexte universitaire, administratif ou communautaire est par le biais du Journal canadien de la médecine d'urgence.

Version imprimée du JCMU : la date butoir pour placer une annonce dans la version imprimée du JCMU est un mois avant la date de parution de la revue. Pour connaître les tarifs pour les annonces et obtenir de plus amples renseignements, veuillez communiquer avec Beverly Kirkpatrick ou Deborah Rodd des Publications de l'AMC (800 663-7336 ou 613 731-8610 x2127 ou x2314, advertising@cma.ca).

\section{À LA RECHERCHE D'UN MÉDECIN D'URGENCE?}

PRIME SPÉCIALE : Les médecins qui placeront une annonce dans la version imprimée du $J C M U$ recevront sans frais un espace pour leur annonce sur le site web de l'ACMU (www.caep.ca).

Site web de l'ACMU seulement : Pour placer une annonce sur le site web de l'ACMU seulement, le coût est de 100 \$ par mois et l'annonce peut être placée en tout temps. Veuillez communiquer avec le siège social de l'ACMU pour les annonces sur le site web seulement (800 463-1158 ou advertising @caep.ca). 